\title{
Memory and identification of simulated odors in elderly and young persons
}

\author{
JOSEPH C. STEVENS, WILLIAM S. CAIN, and ANNICK DEMARQUE \\ John B. Pierce Foundation Laboratory and Yale University, New Haven, Connecticut
}

\begin{abstract}
Twenty elderly and 20 young persons were tested for memory of chemically simulated odors, minutes after inspecting them, 2-3 h later, and 1 week later. They were also asked to identify the odors by name at the end of the experiment. Unlike most of the everyday odors they simulated, the odors could be varied in intensity by liquid dilution. As in an earlier study, odor memory was poorer in elderly than in young subjects. Performance of the elderly fell to chance in 2-3 $\mathrm{h}$. Over the relatively moderate range used, intensity seemed to matter little to memory, but, over the same range, intensity did play a significant, though relatively small, role in identification. On the average, the stronger the odor, the better the identification.
\end{abstract}

Aging is known to affect several different aspects of olfactory functioning adversely: (1) Absolute thresholds rise (for reviews of numerous studies, see Murphy, 1986, and Schiffman, 1979); (2) suprathreshold odor strength declines (Stevens \& Cain, 1986, 1987); (3) adaptation speeds up and recovery from adaptation slows down (Stevens, Cain, Schiet, \& Oatley, 1989); (4) ability to distinguish odor quality falls off (Cain, Reid, \& Stevens, in press; Schiffman \& Leffingwell, 1981); (5) ability to identify common odorants also falls off (Doty et al., 1984; Schemper, Voss, \& Cain, 1981); and finally, (6) it has recently been shown that aging also depresses odor recognition memory (Murphy, Cain, Gilmore, \& Skinner, 1989). The latter investigation involved a paradigm similar to that used in earlier studies of odor memory (e.g., Engen \& Ross, 1973; Lawless \& Cain, 1975). The subjects smelled a set of odors and after various retention intervals, smelled the original odors (targets) interspersed with new odors (distractors), under instructions to state whether each odor was from the original set or was new.

Under this procedure, young persons typically perform well right after original inspection and forget the odors slowly. In one study, for example, even after 2 months subjects were showing correct recognition about $70 \%$ of the time, relative to the chance level of $50 \%$ (Lawless, 1978). In contrast, Murphy et al. (1989) showed that after 2 weeks, elderly subjects' performance had fallen to chance level, whereas even after 6-7 months the young performed well above chance.

An important question arises out of this and earlier findings. Does the strength of the odors play a role in the ability to remember them over time? Is it easier to encode and remember an odor if it is strong? If so, then the inferior performance of the elderly could be put down to

This research was supported by Grant AG04287 from the National Institute on Aging. Correspondence should be addressed to Joseph C. Stevens, John B. Pierce Foundation Laboratory, 290 Congress Ave., New Haven, CT 06519. their known loss of suprathreshold odor strength. If not, then the results would seem explainable in terms of the types of encoding actually employed rather than the mere ability to encode.

A similar question can be asked about the ability to identify odors by labeling them. When one gets a strong whiff of an odor, can one label it better for what it is? Performance on odor identification to screen for hyposmia (reduction in olfactory ability) suggests that this should be so. Even persons with mild hyposmia-judged by modestly elevated detection thresholds-can identify fewer odors than persons with normal smell (Cain, 1989; Cain \& Gent, 1986). Nevertheless, deliberate manipulation of suprathreshold odor intensity has yet to demonstrate the connection between intensity and identifiability. In the present study, we sought first-order answers to these questions, using simulated odors that could be varied in intensity by liquid dilution.

\section{METHOD}

\section{Subjects}

Twenty young subjects (19-33 years, mean 22.3), 8 women and 12 men, and 20 elderly subjects (61-88 years, mean 73.4), 17 women and 3 men, served in three test sessions each. The young were tested in the laboratory, the elderly at a senior citizens' center in New Haven. The elderly all lived independently at home. On a scale from "good" to "very poor" health, one young subject rated herself "average," all the others "good." Of the elderly subjects, 3 said "average," all the rest "good."

\begin{abstract}
Materials
Twenty-five odorants were used, furnished by International Flavors and Fragrances, Inc. These are listed in Table 1 by chemical name, as well as by the common names of everyday odorants they simulate. The reason for using simulated odors, rather than common object odorants, is that it would be difficult or impossible to dilute the latter to known concentrations in order to study the effect of odor strength on odor memory and identification. Each odorant was diluted with mineral oil to provide weak, medium, and strong odor levels, whose concentrations are also listed in Table 1, thereby providing 75 stimuli in all. An attempt was made by a laboratory assistant and one of the authors (Cain) to equate the odorants for strength at three levels. In addition, the weak odorant strength was chosen to match the strength of $n$-butanol at a vapor-
\end{abstract}


Table 1

Odorants Used to Study Odor Memory and Odor Identification

\begin{tabular}{llccc}
\hline \multicolumn{1}{c}{ Simulated } & \multicolumn{1}{c}{ Chemical Name } & Weak & Medium & Strong \\
\hline apple & verdox & 0.07 & 0.43 & 2.58 \\
banana & amyl acetate & 0.03 & 0.20 & 1.21 \\
bell pepper & galbazine 1\% dep & 0.01 & 0.06 & 0.34 \\
carnation & iso eugenol & 0.02 & 0.11 & 0.66 \\
cedarwood & vertofix coeur & 0.12 & 0.69 & 4.17 \\
cherry & benzaldehyde FFC & 0.03 & 0.17 & 1.01 \\
chicken fat & 2,4-decadienal & 0.03 & 0.17 & 1.01 \\
cinnamon & cinnamic aldehyde & 0.03 & 0.17 & 1.01 \\
citronella oil & citronellal FH & 0.03 & 0.17 & 1.01 \\
cloves & eugenol \#1 & 0.03 & 0.17 & 1.01 \\
cucumber & nonadienal diethyl acetal & 0.03 & 0.17 & 1.01 \\
cumin & cumin nitrile & 0.02 & 0.11 & 0.66 \\
gardenia & styrallyl acetate coeur & 0.02 & 0.11 & 0.66 \\
grass & cis-3-hexanol & 0.01 & 0.09 & 0.52 \\
hyacinth & hyacinth body & 0.03 & 0.17 & 1.01 \\
jasmine & hexyl cinnamic aldehyde coeur & 0.12 & 0.69 & 4.17 \\
licorice & methyl chavicol coeur & 0.03 & 0.17 & 1.01 \\
mushroom & amyl vinyl carbinol & 0.02 & 0.11 & 0.66 \\
peach & peach aldehyde coeur & 0.02 & 0.11 & 0.66 \\
pine needles & iso bornyl acetate & 0.02 & 0.11 & 0.66 \\
popcorn & popcorn chemical 1\% & 0.01 & 0.06 & 0.34 \\
rose & citronellol coeur & 0.02 & 0.11 & 0.66 \\
strawberry & aldehyde C16 \#2 & 0.02 & 0.11 & 0.66 \\
violet & methyl ionone gamma coeur & 0.02 & 0.11 & 0.66 \\
wintergreen & methyl salicylate & 0.03 & 0.17 & 1.01 \\
\hline Not & m & &
\end{tabular}

Note-The concentrations are the percentages of the stock odor $(99.9 \%$ pure) after dilution with mineral oil.

phase concentration of $9 \mathrm{ppm}(\mathrm{v} / \mathrm{v})$, which was reliably smelled by all the subjects in a screening test before the main experiment. Matching so many odors is no easy task, but by trial and error we got reasonably good sets. In any case, precision matching was not crucial to the experimental design.

All odors throughout the experiment were presented from 240-ml plastic "shampoo" bottles (Monsanto). The subjects placed the spout just below the nose and squeezed the bottle first below one nostril, and then below the other, while sniffing.

The first test session began with sensitivity screening to excuse from further testing persons too hyposmic to partake meaningfully in an experiment on odor memory. Based on much experience with the compound $n$-butanol, we placed the cutoff at a concentration level of $9 \mathrm{ppm}$. The subject was first required to decide five times which of two bottles contained the odorant (at $3 \mathrm{ppm}$ ) as opposed to solvent only (deionized $\mathrm{HOH}$ ). If correct on all five trials, the subject went on to another five trials at $9 \mathrm{ppm}$ versus solvent only. If correct on all of these trials, the subject was accepted for further testing. If a subject erred one or more times at $3 \mathrm{ppm}$, then seven consecutive correct choices were required at $9 \mathrm{ppm}$ for the subject to qualify for further testing. Under these rules, only 1 elderly person and 1 young person had to be excused.

The subject then smelled in succession 12 odorants from the list in Table 1, at intervals of $30 \mathrm{sec}$. This we called the inspection set, and it varied across subjects in terms of the compounds selected to make it up. The subjects were instructed that they should try to remember the odorants and that they would be tested later to see how well they could remember them. The 12-item inspection set consisted of 4 weak, 4 medium, and 4 strong stimuli. For any given subject, the remaining 13 odorants in the total set of 25 provided a library of 39 (13 odorants $X$ three levels) from which to select stimuli to serve as distractors to be discriminated from the inspection odorants in subsequent memory tests; however, none of the distractors chosen ever consisted of another level of any of the 12 target odorants. Each memory test employed the targets (i.e., the same odorants at the same concentration levels) and 12 distractors, 4 of which were weak, 4 medium, and 4 strong.

The first of three memory (recognition) tests took place right after initial inspection. For the 24 stimuli, presented in random order, the subject judged whether the odorant was "old" (i.e., a target from the inspection set) or "new." The first test session, including instructions, sensitivity screening, inspection, and recognition, took about $30 \mathrm{~min}$, and was always held in the morning.

The second test was held 2-3 h later, in the afternoon. The distractors used in the test consisted of the same compounds that had been used in the first test but at different concentration levels; that is, a given chemical at a strong level in the first recognition test would be presented at a medium or weak level in the second test. The same principle held for the third test, so that each distractor was presented at all three levels in the course of the three tests. The second test took about $15 \mathrm{~min}$.

The third test took place 7 (occasionally 6 or 8) days later. The task was the same as before. After completion, the targets were presented again one by one for identification. If the subject was unable to name the odor correctly without help (unprompted identification), a card was displayed containing four labels (e.g., baby powder, cucumber [targeted odor], cat food, peanut butter) from which the subject tried to select the correct label (prompted identification). The third session lasted about $30 \mathrm{~min}$. Note from Table 1 that several of the odorants had a floral quality that could make exact identification difficult. For this reason, when the target odorant was floral, the other three labels on the card were chosen to be nonfloral in quality.

\section{RESULTS}

The measure $A^{\prime}$, closely related to $d^{\prime}$, served as the index of memory. $A^{\prime}$ is a nonparametric statistic and thus circumvents assumptions concerning the form of the memory operating-characteristic curve (Pollack \& Norman, 1964). It is computed with the following formula:

$A^{\prime}=0.5+[(H-F A)(1+H-F A)] /(4+H)(1-F A)$,

where $H$ is the proportion of hits for each subject (i.e., correct choice of a target odor) and $F A$ is the proportion of false alarms (i.e., choice of a distractor odor as a target). An $A^{\prime}$ of 1.0 would mean perfect discrimination of 
target and distractor odors; an $A^{\prime}$ of .5 would mean chance performance.

Figure 1 shows how mean $A^{\prime}$ varied with retention interval, for the young and elderly separately but across all three odor strengths. Here can be seen how much the young's performance surpassed the elderly's throughout. Already right after inspection, a large difference emerged. An ANOVA showed significant effects of age $[F(1,38)=$ $32.5, p<.001]$ and retention interval $[F(2,76)=9.8$, $p<.0002]$, but no significant interaction $[F(2,76)=2.03$, $p=.14]$. As gauged by $t$ tests, the elderly's $A^{\prime}$ was significantly above chance in the first test $[t(19)=4.60$, $p<.0001$ ] and last test $(t=2.45, p<.012)$, but not in the test 2-3 $\mathrm{h}$ after inspection $(t=1.09, p=.15)-$ why this was so is hard to say, but it appears that overall the elderly did show some, albeit weak, durable memory for the targets.

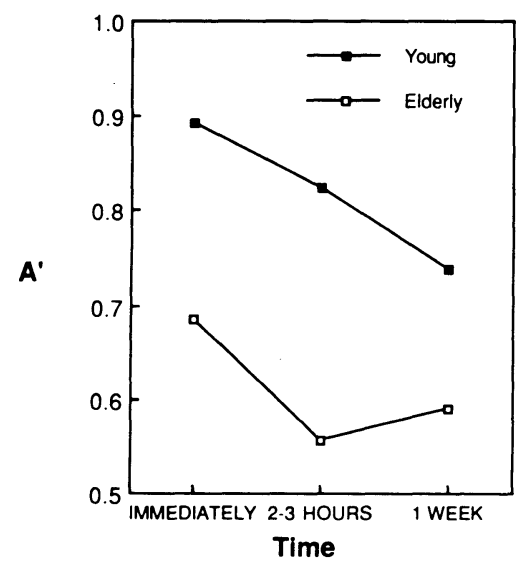

Figure 1. Mean $A^{\prime}$ values for young and elderly for the three recognition tests (collapsed across the three intensities). The spacing on the time axis is arbitrary.

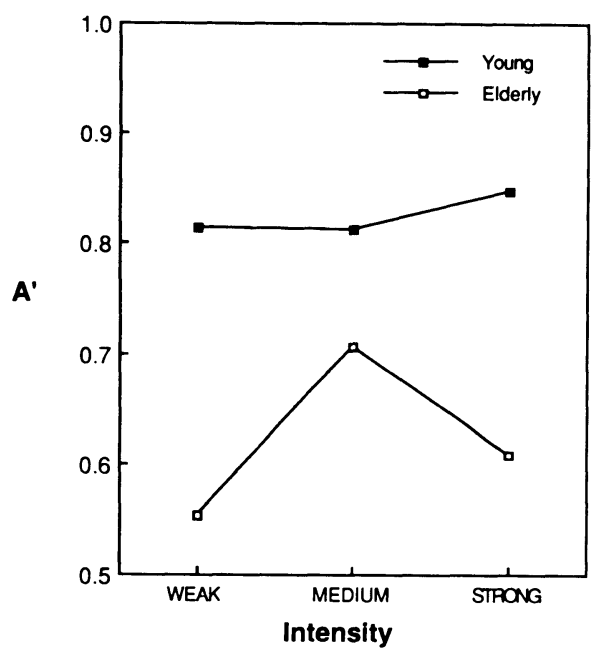

Figure 2. Mean $A^{\prime}$ values for young and elderly at three intensities (collapsed across the three recognition tests).

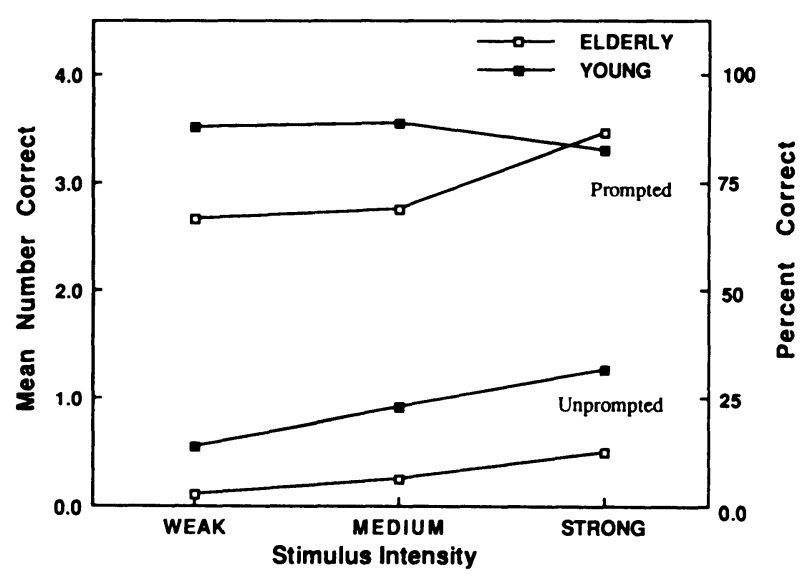

Figure 3. Mean number and percent of correct identifications for young and elderly, prompted and unprompted, for each of three odor intensities.

Figure 2 shows how mean $A^{\prime}$, collapsed across test times, varied with odor strength. An ANOVA again showed a significant effect of age $[F(1,38)=32.8$, $p<.001]$ but not of odor strength $[F(2,76)=2.02, p=$ .14], with a marginally significant interaction of intensity $X$ age $[F(2,76)=2.53, p=.087]$. It is evident from Figure 2 that for the elderly $A^{\prime}$ may be lower for the weak odorants than for the medium and strong. Indeed, performance on the weak odorants failed to exceed chance $(t=$ $0.99, p<.17$ ) but on the strong odorants approached significance $(t=1.65, p<.057)$ and on the medium level achieved significance $(t=6.54, p<.0001)$. In fact, at the low level, despite the screening for sensitivity at the beginning of the first session, many elderly subjects complained that they could smell nothing.

Figure 3 shows both prompted and unprompted scores on the identification test. An ANOVA revealed a significant effect of age $[F(1,38)=16.58, p<.0002]$, of prompting $[F(1,38)=984, p<.0001]$, and of odor strength $[F(2,76)=5.96, p<.004]$. With prompting, the elderly performed as well as the young on the strong odors.

\section{DISCUSSION}

As in several earlier studies, odor memory of young subjects was good immediately after inspection, and it declined slowly, so that even after a week recognition was still considerable. In fact, it was substantially better than the elderly's recognition only minutes after initial inspection. After 2-3 h, the elderly showed little, if any, recognition; on the average, they judged correctly only 13.25 times out of 24 , as compared with 15.3 times in the first test. In contrast, the young chose correctly 20 times in the first test, which declined to 16.5 times a week later.

Although the results agree with the earlier finding that aging impairs odor recognition memory, both our young and elderly performed somewhat worse than the respective young and elderly studied by Murphy et al. (1989). The difference may have to do with their use of everyday common substances rather than simulated odors, which are, after all, imperfect olfactory copies. It has been established that good identifiability favors recognition (Rabin \& Cain, 1984). Also, familiarity favors recognition. Perusal of Table 1 reveals several odors that might be quite unfamiliar to many persons. 
More than one factor may account for the difference here between the young and the elderly. The elderly claimed particular difficulty with weak targets and distractors. This raises the possibility that the attempt to match all the odorants to $9 \mathrm{ppm}$ of $\boldsymbol{n}$-butanol may have been off enough to have put some of the weak odorants below some subjects' thresholds. We may also note that the elderly have been found to adapt faster to odorants and to recover sensitivity after adaptation more slowly than the young (Stevens et al., 1989). Thus, it may be that strong odors rendered the weak ones insensible to at least some of the elderly subjects.

Such explanations take the claims of the elderly about apparent intensity at face value. Perhaps in an attempt to rationalize their poor performance some elderly seized upon intensity as a key. Despite their claims, however, they performed little better with odorants over 30 times more concentrated than the weak level. In general, thresholds of the elderly lie up to only an order of magnitude above those of the young (Cain \& Stevens, 1989). Even with unscreened participants, therefore, a factor of 30 would seem sufficient to overcome any differences in sensitivity. If threshold sensitivity alone governed the difference in recognition memory between the young and the elderly, then the elderly should have performed at least as well at the highest concentration as the young did at the lowest. The elderly fell far short in that respect.

Aside from the matter of sensory detection, intensity could control the distinctiveness, and hence discriminability, of stimuli. Distinctiveness could presumably aid encoding of perceptual characteristics directly and semantic encoding indirectly. Regarding the latter, a more distinct stimulus will often prove more identifiable and, as Rabin and Cain (1984) demonstrated, more recognizable. In the present case, intensity aided identification; but the rate of identification fell well below that found with real-world items, and the degree to which identification increased with intensity proved rather small. Whereas young people can normally identify (unprompted) about half of everyday items (see, e.g., Cain, 1979), they could identify only $23 \%$ of our simulated odors. The elderly exhibited a commensurate reduction in their customarily poorer ability to identify (Schemper et al., 1981). Such poor identification meant that neither group had a typical opportunity to employ the semantic encoding associated with identifiable odors. Insofar as semantic encoding largely mediates odor recognition memory (cf. Lyman \& McDaniel, 1986, and Walk \& Johns, 1984), a small increase in identification would presumably at most lead to a small increase in recognition at most.

Our finding that odor recognition memory in the elderly may slip from moderately good to chance level finds a precedent in the data of Murphy et al. (1989). They found odor recognition to survive for an hour but to fall to chance by 2 weeks. In accompanying control tasks that required graphic memory, their elderly performed generally as well as their young out to 6 months, at which point both groups still performed above chance. Hence, the difference in recognition memory proved strictly olfaction-specific.

Without tests in the interval between $1 \mathrm{~h}$ and 2 weeks, Murphy et al. (1989) had no way to tell when odor recognition would fall to chance. It appears that the loss occurs sooner $(2-3 \mathrm{~h})$ rather than later (1 week) in that interval. Why such a precipitous drop should occur is a mystery, still more so in view of the common finding that the elderly typically show little or no deterioration of recognition memory across a variety of tasks. In the present case, performance of the elderly after 1 week seemed to show some recovery. Since the young gave no indication of any such reminiscence, or hypermnesia, we doubt its importance. Although rarely demonstrated, hypermnesia can occur in recognition tasks (Erdelyi, 1984).

\section{REFERENCES}

CaIN, W. S. (1979). To know with the nose: Keys to odor identification. Science, 203, 467-470.

CAIN, W. S. (1989). Testing olfaction in a clinical setting. Ear, Nose \& Throat Journal, 68, 316-328.
CAIN, W. S., \& GENT, J. F. (1986). Use of odor identification in clinical testing of olfaction. In H. L. Meiselman \& R. S. Rivlin (Eds.), Clinical measurement of taste and smell (pp. 170-186). New York: Macmillan.

Cain, W. S., \& Stevens, J. C. (1989). Uniformity of olfactory loss in aging. In C. Murphy, W. S. Cain, \& D. M. Higsted (Eds.), Nutrition and the chemical senses in aging: Recent advances and current research needs. Annals of the New York Academy of Sciences, 561, 29-38.

Cain, W. S., Reid, F., \& Stevens, J. C. (in press). Missing ingredients: Aging and the discrimination of flavor. Journal of Nutrition for the Elderly.

Doty, R. L., Shaman, P., Applebaum, B. L., Giberson, R., SikSORSKI, L., \& RoSENBERG, L. (1984). Smell identification ability: Changes with age. Science, 226, 1441-1443.

ENGEN, T., \& Ross, B. (1973). Long term memory of odors with and without verbal descriptions. Journal of Experimental Psychology, 100, 221-227.

ERDELYI, M. (1984). The recovery of unconscious (inaccessible) memories: Laboratory studies of hypermnesia. In G. H. Bower (Ed.), The psychology of learning and memory (Vol. 18, pp. 95-127). Orlando, FL: Academic Press.

LAWLESS, H. T. (1978). Recognition of common odors, pictures, and simple shapes. Perception \& Psychophysics, 24, 493-495.

LAWLESS, H. T., \& CaIN, W. S. (1975). Recognition memory for odors. Chemical Senses \& Flavor, 1, 331-337.

Lyman, B. J., \& McDANIEL, M. A. (1986). Effects of encoding strategy on long-term memory for odours. Quarterly Journal of Experimental Psychology, 38, 753-765.

MuRPhy, C. (1986). Taste and smell in the elderly. In H. L. Meiselman \& R. S. Rivlin (Eds.), Clinical measurement of taste and smell (pp. 343-371). New York: Macmillan.

MurPhy, C., Cain, W. S., Gilmore, M. M., \& SkinNer, R. B. (1989). Aging impairs recognition memory for odors. Manuscript submitted for publication.

Pollack, I., \& Norman, D. A. (1964). A non-parametric analysis of recognition experiments. Psychonomic Science, 1, 125-126.

Rabin, M. D., \& CAIN, W. S. (1984). Odor recognition: Familiarity, identifiability, and encoding consistency. Journal of Experimental Psychology: Learning, Memory, \& Cognition, 10, 316-325.

SChemper, T., Voss, S., \& CaIN, W. S. (1981). Odor identification in young and elderly persons: Sensory and cognitive limitations. Journal of Gerontology, 36, 446-452.

Schiffman, S. (1979). Changes in taste and smell with age: Psychophysical aspects. In J. M. Ordy \& K. Brizzee (Eds.), Sensory systems and communication in the elderly: Vol. 10. Aging (pp. 227-246). New York: Raven Press.

Schiffman, S., \& LefFingwell, J. C. (1981). Perception of odors of simple pyrazines by young and elderly subjects: A multidimensional analysis. Pharmacology, Biochemistry, \& Behavior, 14, 787-798.

Stevens, J. C., \& CaIN, W. S. (1986). Smelling via the mouth: Effect of aging. Perception \& Psychophysics, 40, 142-146.

Stevens, J. C., \& CaIN, W. S. (1987). Old-age deficits in the sense of smell as gauged by thresholds, magnitude matching, and odor identification. Psychology \& Aging, 2, 36-42.

Stevens, J. C., Cain, W. S., Schiet, F. T., \& Oatley, M. W. (1989). Olfactory adaptation in old age. Perception, 18, 265-275.

WALK, H. A., \& JoHNS, E. E. (1984). Interference and facilitation in short-term memory for odors. Perception \& Psychophysics, 36, 508-514. 